\title{
Article \\ Research of Interaction between Ultra-Short Ultra-Intense Laser Pulses and Multiple Plasma Layers
}

\author{
Fang Feng ${ }^{1,2}$ and Gang Lei ${ }^{3, *}$ \\ 1 College of Arts and Sciences, Northeast Agricultural University, Harbin 150030, China; fengfang@neau.edu.cn \\ 2 Key Laboratory of Icing and Anti/De-Icing, China Aerodynamics Research and Development Center, \\ Mianyang 621000, China \\ 3 Rocket Force University of Engineering, Xi' an 710025, China \\ * Correspondence: Leig603@163.com
}

check for updates

Citation: Feng, F.; Lei, G. Research of Interaction between Ultra-Short Ultra-Intense Laser Pulses and Multiple Plasma Layers. Symmetry 2021, 13, 1175. https://doi.org/ $10.3390 /$ sym 13071175

Academic Editor:

Alexander Shapovalov

Received: 19 May 2021

Accepted: 21 June 2021

Published: 29 June 2021

Publisher's Note: MDPI stays neutral with regard to jurisdictional claims in published maps and institutional affiliations.

Copyright: (c) 2021 by the authors. Licensee MDPI, Basel, Switzerland. This article is an open access article distributed under the terms and conditions of the Creative Commons Attribution (CC BY) license (https:// creativecommons.org/licenses/by/ $4.0 /)$.

\begin{abstract}
In this research, we studied the interaction between the ultra-intense laser and multiple copper layers covered with multiple hydrogen layers. The research conditions are based on the symmetric and asymmetric structure of multilayer copper and hydrogen. It was found that the acceleration obtained from the first copper and hydrogen layer plasma was higher and occurred earlier than the second copper and hydrogen layer plasma. We investigated the spatial distribution and phase-space distribution of copper electrons, copper ions, hydrogen electrons, and hydrogen protons with different widths of the front hydrogen layer and the front copper layer, respectively. Theoretical simulations show that when the ultra-intense laser was irradiated in multiple copper layers coated with multiple hydrogen layers targets, some plasma phase-space distribution varied clearly in the different thicknesses of the first hydrogen layer or first copper layer, while some plasma were not influenced by the thickness of these two layers.
\end{abstract}

Keywords: acceleration effect; plasma; copper layers; hydrogen layer; ultra-intense laser

\section{Introduction}

Laser-driven plasma electron acceleration has obtained wide consideration due to the potential for fabricating small-scale accelerators [1]. In 2002, A. Pukhov observed the laser wake with the shape of a solitary plasma cavity by observing ultra-short electron bunches that emerged from laser wake fields driven over the wave-breaking threshold via few-cycle laser pulses shorter than the plasma wavelength by simulation [2]. Then, two experiments were reported in 2004. One was to drive a plasma bubble that traps and accelerates plasma electrons within $3 \mathrm{~mm}$ length by laser [3]. Another was the application of a preformed plasma density channel to guide a relativistically intense laser, resulting in longer propagation distance in this research [4]. The research of the interaction of an intense femtosecond laser with over-dense foil by particle-in-cell simulations was performed in 2004 [5]. High-energy laser pulses and different density gradient plasma interactions were researched by J. Wolowski in 2005, investigating the properties of laser-created ion streams performed under the presence of pre-pulses of various parameters [6]. In addition, the self-focusing phenomenon with extremely high-intensity ps or ns laser pulses and plasma interactions were investigated by H. Hora et al. [7]. In 2012, ultra-short, ultraintense laser interaction with the uniform and parabolic plasma density profiles were simulated and analyzed, which showed that a parabolic plasma density profile can provide better guidance for the ultra-short and ultra-intense pulse. They also found self-guiding can be realized by relativistic self-focusing in uniform plasma at higher densities. This can simplify the experiment to produce more accelerated electrons [8]. The interactions between lasers and different plasma are carried out in different groups. The L-shell X-ray emission measurement of Au plasma produced by an intense femtosecond laser experiment was conducted by Yang Zhaorui et al. in 2012 [9]. The ion acceleration that occurred 
during the interaction of ultra-short laser pulses with under-dense helium plasma was researched by A. Lifschitz's group [10]. A study of ion heating in CD2-A1-CD2 sandwich targets irradiated by an ultra-short laser was reported in 2013. They found that Al-CD2 interface pressure gradients pushed deuteron ions to the target outer regions [11]. In 2017, Dragos Tatomirescu et al. studied the characteristics of an accelerated ion beam while changing the target parameters with constant laser pulse parameters. They studied the spatial distribution of particle beams, maximum particle energy, etc. They also studied the impacts of the curvature target coupled with a cone laser focusing structure [12,13]. The experimental results of the generation of highly collimated electron beams with a few degrees divergence angle, mega-electron-volt level non-thermal spectra peak by the interaction of powerful sub-picosecond laser pulses, and solid target in grazing incidences were reported by Yong Ma et al. in 2018 [14]. The theoretical results of the interaction of short intense laser pulses and diluted plasma, the plasma wave formation, wave-breaking, and slingshot effect were reported by the Gaetano Fiore group in 2018 [15]. In 2019, W.J. Ma reported the interaction between ultra-intense femtosecond laser pulses and double-layer targets composed with under-dense plasma and ultra-thin foils. The generation of highly energetic carbon ions up to $48 \mathrm{MeV}$ per nucleon was observed [16].

In this paper, we investigated theoretical simulations of the interaction between the ultra-intense laser pulses and multiple copper layers coated with multiple hydrogen layers. The research conditions are based on the symmetry and asymmetry structures of multilayer copper and Hydrogen. Simulation results show that the first copper and hydrogen layer plasma acceleration attained was higher and occurred earlier compared with the second copper and hydrogen layer plasma. Besides, the spatial distribution and the phase-space distribution of copper electrons, copper ions, hydrogen electrons, and hydrogen protons in differing thicknesses of the respective first hydrogen and copper layers are presented and discussed. We found that various thicknesses of the first hydrogen or copper layer played an important role in the varying spatial distribution and phase-space distribution of some plasma. Meanwhile, other plasma spatial distributions and phase-space distributions did not change under the various thicknesses of the first hydrogen or copper layers.

\section{Theory}

The laser and plasma interaction is closely related to the laser parameters, the material properties, and state parameters of the target plasma. Among them, the most decisive factor is the laser intensity and the density of the plasma. The laser intensity is $I_{0}=\frac{E_{0}}{S \tau}$, where $E_{0}$ is the laser power, $S$ denotes the Laser irradiation area, and $\tau$ is the FWHM of the laser pulse. Laser amplitude is $a_{0}=\frac{e A_{0}}{m_{e} c^{2}}$, where $A_{0}$ is the laser pulse peak value, $m_{e}$ represents the electron quality, $c$ is the light speed, and $e$ is the electron quantity. For linear polarization lasers, $A_{0}=\sqrt{\frac{2 I_{0} \lambda_{0}^{2}}{\pi c}}$ and circular polarization lasers $A_{0}=\sqrt{\frac{I_{0} \lambda_{0}^{2}}{\pi c}}$, where $\lambda_{0}$ is the laser wavelength. The electromagnetic field cannot propagate in plasma with densities over a certain threshold. This threshold density is: $n_{c}=\frac{\pi m_{e} c^{2}}{e^{2} \lambda_{0}^{2}}$. In this simulation, the electric field spreading along the $x$-direction is $E=A \times \operatorname{profile}(y, z) \times e n v(x, t) \times \sin (\omega t-k t)$, where profile and $e n v$ represent the $y$-direction, $z$-direction, $x$-direction, and laser envelope shape respectively. $A$ is the laser pulse peak value, $\omega$ is the angular frequency of the laser wave, and $k$ is the wave number.

In the process of the interaction of ultra-short, ultra-intense laser pulses and plasma, the laser field accelerates the ions. We use the Vsim PIC software program to simulate the interaction of laser pulses and multiple plasma layers. We set the dimensionless quantity laser amplitude to $1 \times 10^{13}$, the laser wavelength to $1 \times 10^{-6}, \mathrm{Cu}$ and $\mathrm{H}$ ion densities to $1 \times 10^{27}$, and so on. Under the interaction of this strong laser field, the relativity effect generates the ponderomotive force to push electrons and form a strong electrostatic field on the back surface of the target. The force can be written as $F=-m_{0} c^{2} \nabla \gamma$, where $\gamma$ is the relativistic factor. From this function, it is notable that electrons get more acceleration than protons because the proton's quality is larger than the electron's quality [17]. For the 
ion simulations, the first step is to initialize the ion's position and velocity. The second step is using the average approximate method to get the charge density and current density distribution. Then, by using Maxwell functions: $\nabla \cdot \boldsymbol{E}=4 \pi \rho, \nabla \cdot \boldsymbol{B}=0, \nabla \times \boldsymbol{E}=$ $-\frac{\partial B}{C \times \partial t}, \nabla \times B=\frac{1}{c}\left(\frac{\partial E}{\partial t}+4 \pi J\right)$ to get the electric and magnetic fields, where $E$ is the electric field, $B$ is the magnetic field, $J$ is the current density, and $\rho$ is the charge density. So, we can calculate the electromagnetic force on every particle. The position and velocity of each particle at the next moment can be calculated by the motion function: $\frac{d \boldsymbol{P}}{d t}=q\left(\boldsymbol{E}+\frac{v \times \boldsymbol{B}}{c}\right)$, where $\boldsymbol{P}$ is the momentum, $v$ is the velocity of the particle, $q$ is the particle electric charge, and $\mathrm{c}$ is the speed of light [18].

\section{Simulation Results of The Spatial Distribution of Particles}

We studied the interaction between ultra-short, ultra-intense lasers and multiple hydrogen layers covered on multiple copper layers. The hydrogen layer and the Copper layer are symmetrically distributed. The simulation results under hydrogen layers with $1 \times 10^{-6} \mathrm{~m}$ width and copper layers with $5 \times 10^{-6} \mathrm{~m}$ width are shown in Figure 1 . Small pieces were hydrogen layers, the dark green was hydrogen protons, blue was hydrogen electrons, light green was copper electrons, and red was copper ions. From Figure 1b,c, we can observe that the electromagnetic field was reflected after hitting the target. The hydrogen electrons accelerated to spread in the copper. The hydrogen protons also accelerated to move in copper. Figure 1d,e shows that the first hydrogen layer's electrons were reflected when they hit the second hydrogen layer, pushing the second hydrogen layer's electrons to spread into the second copper layer. The first hydrogen layer's protons gained acceleration to the right and left, while the second hydrogen layer's protons did not gain acceleration. It should be noted that electrons moved faster than the protons since electrons are lighter than the protons. Figure $1 \mathrm{~d}-\mathrm{g}$ shows that the copper electrons spread widely after being hit. Figure 1a-g time steps numbers are 9000, 13,000, 16,000, 19,000, 22,000, 29,000, and 39,000 , respectively.

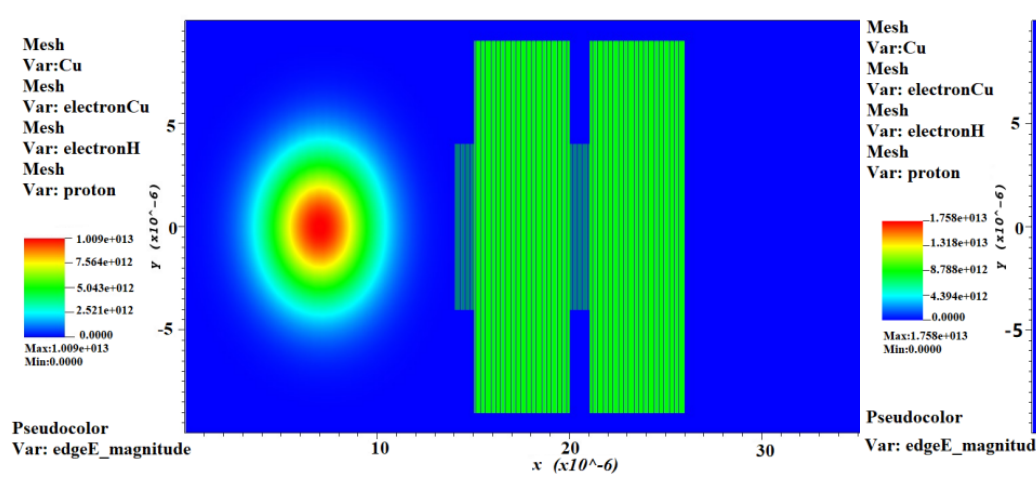

(a)

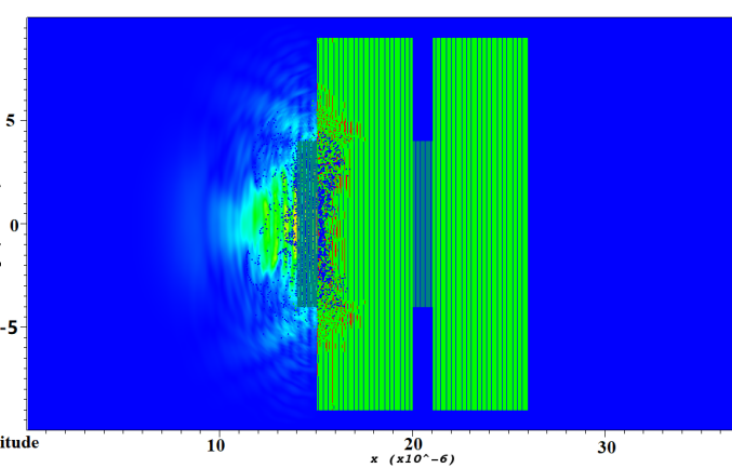

(b)

Figure 1. Cont. 


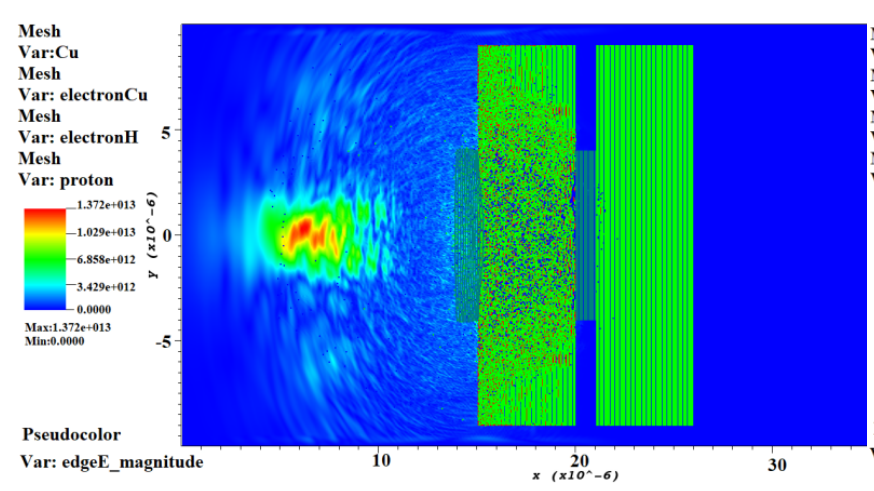

(c)

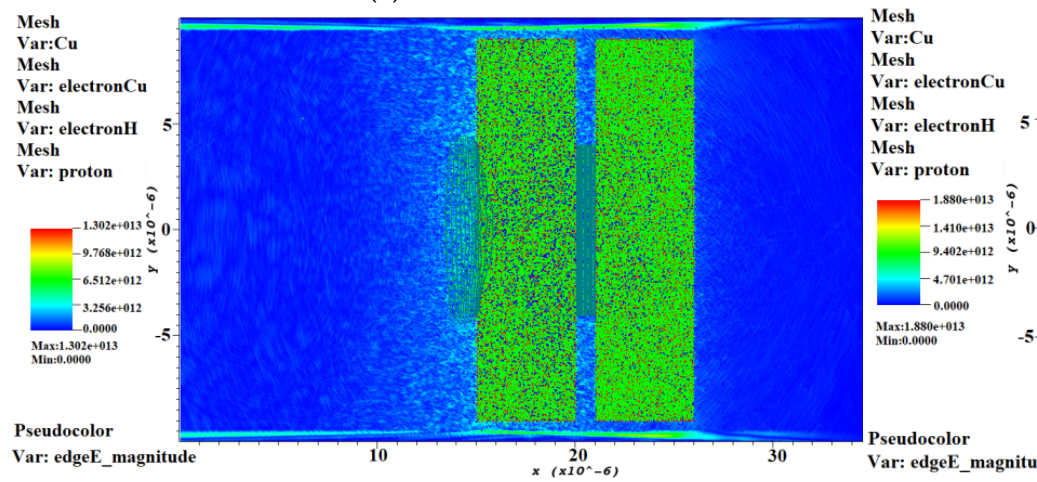

(e)

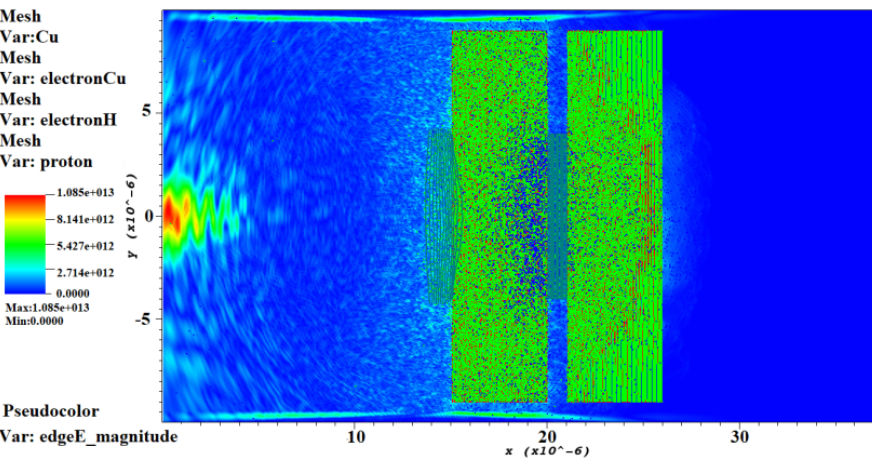

(d)

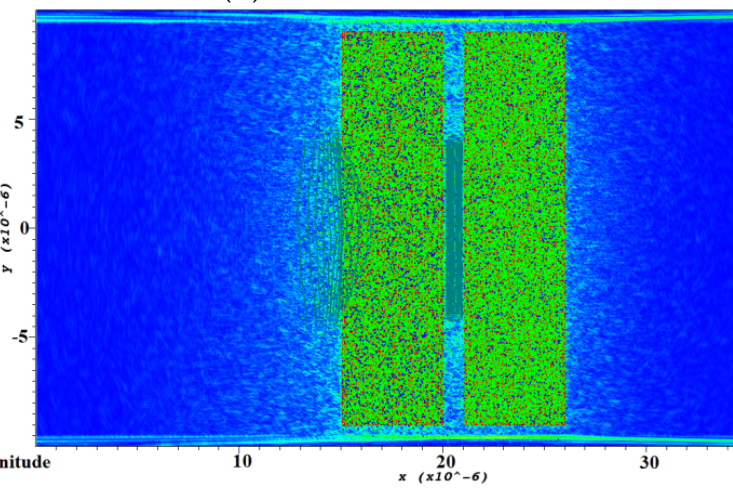

(f)

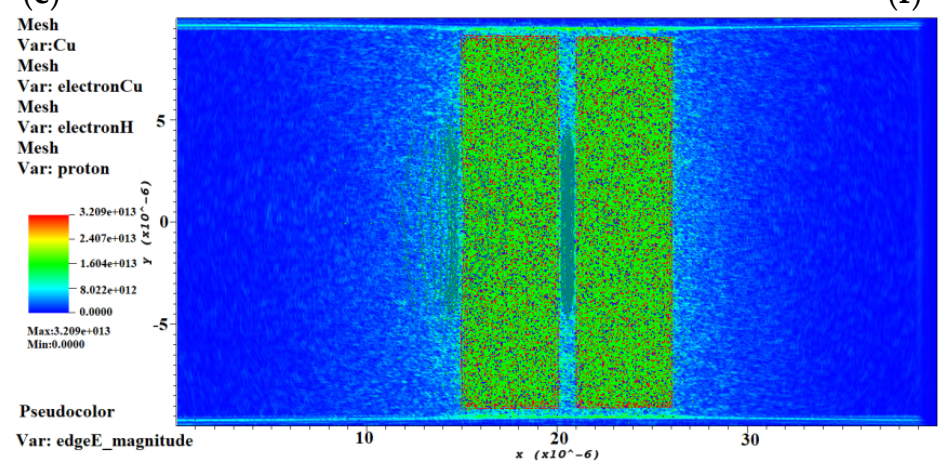

$(\mathrm{g})$

Figure 1. The interaction of ultra-short, ultra-intense laser pulses with multiple hydrogen and copper layers. The light green is copper electrons, the red is copper ions, the blue is hydrogen electrons, and the dark green is hydrogen protons. (a-g) show the movement of particles over time with the time steps numbers being 9000, 13,000, 16,000, 19,000, 22,000, 29,000 , and 39,000, respectively.

\section{Numerical Simulation Results of Particle Phase-Space Distribution}

We investigated the phase-space distribution of hydrogen protons following the time as shown in Figure 2. Figure 2a-f time steps numbers are 0, 12,000, 16,000, 24,000, 32,000, and 40,000, respectively. The simulation results showed that the acceleration of the first layer of hydrogen protons was increased in the vertical direction at the beginning, which then started to spread in both left and right horizontal directions. It was observed that the second layer of hydrogen protons began to diffuse when the first layer of hydrogen electrons hit the second hydrogen layer. 

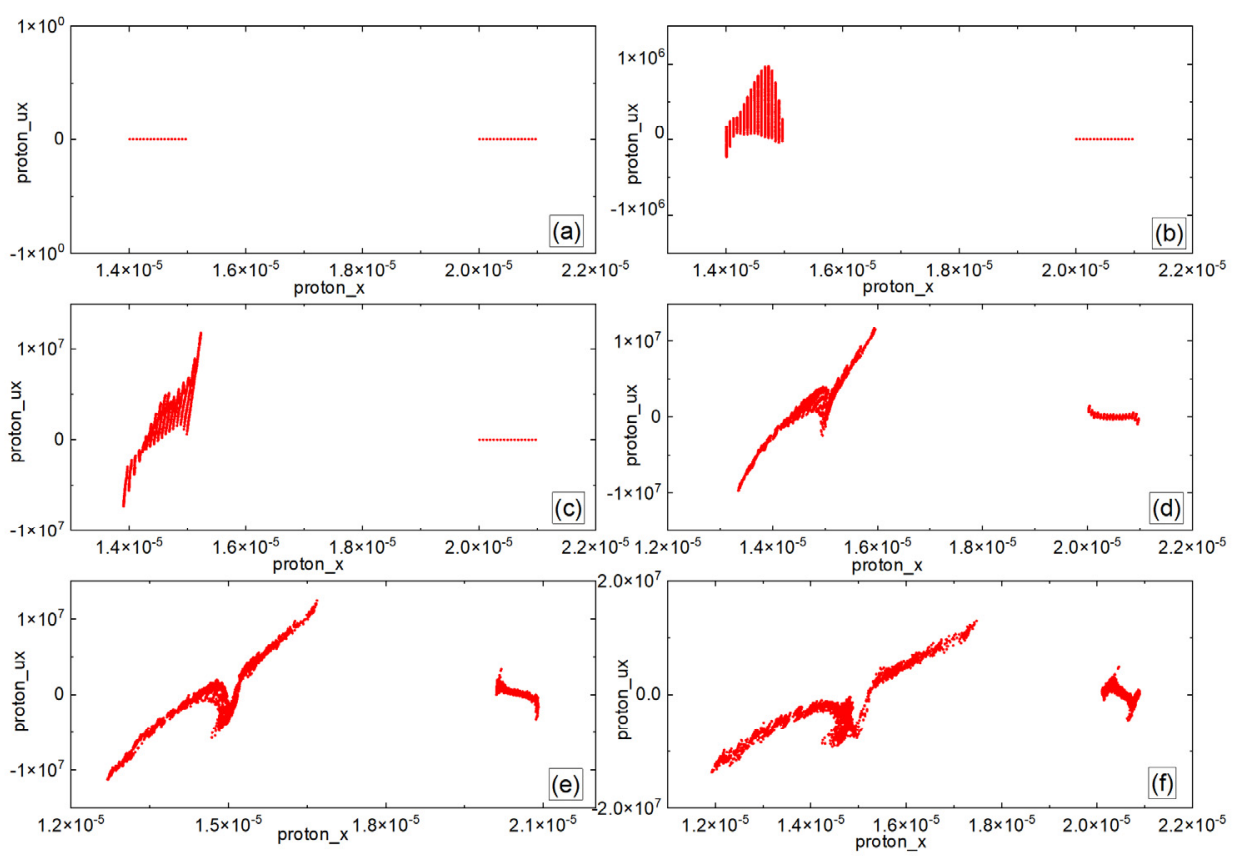

Figure 2. Two layers of hydrogen proton phase-space distribution over time with the time step numbers being 0 (a), 12,000 (b), 16,000 (c), 24,000 (d), 32,000 (e), and 40,000 (f), respectively.

The phase-space distribution of two layers of hydrogen electrons changing with time is displayed in Figure 3. The time steps numbers of Figure 3a-h are 0, 11,000, 15,000, 18,000, $20,000,26,000,34,000$, and 40,000, respectively. From Figure $3 a-h$ it can be noted that an extremely high acceleration of the first layer of hydrogen electrons was obtained, diffusing to the right and moving into copper. When the first layer of hydrogen electrons hit the second hydrogen layer, the first layer passed over the second layer and pushed the second layer to move left due to the high acceleration of the first layer. From Figure $3 \mathrm{f}-\mathrm{h}$, it is notable that the left column is the second layer of hydrogen electrons and the right column denotes the first layer of hydrogen electrons.

We also present a theoretical simulation investigation of the copper proton and electron phase-space distribution. The phase-space distribution of copper ions changing with time is shown in Figure 4. And from Figure 4a-f, the time steps numbers are 0, 8000, 16,000, $24,000,32,000$, and 40,000 , respectively. It can be noted that the first layer of copper ions obtained vertical acceleration in the beginning. After a while, copper ions achieved positive velocity at the end of the first copper layer. Then, the beginning and end parts of the second layer of copper also obtained vertical acceleration. In Figure 5, we illustrate the evolution of the phase-space distribution of copper electrons. As shown in Figure 5, the first layer of copper electrons obtained vertical acceleration first; subsequently, the vertical acceleration of the second layer of copper electrons was achieved. From Figure $5 \mathrm{a}-\mathrm{f}$, the time steps numbers are $0,12,000,16,000,20,000,27,000$, and 39,000, respectively. 

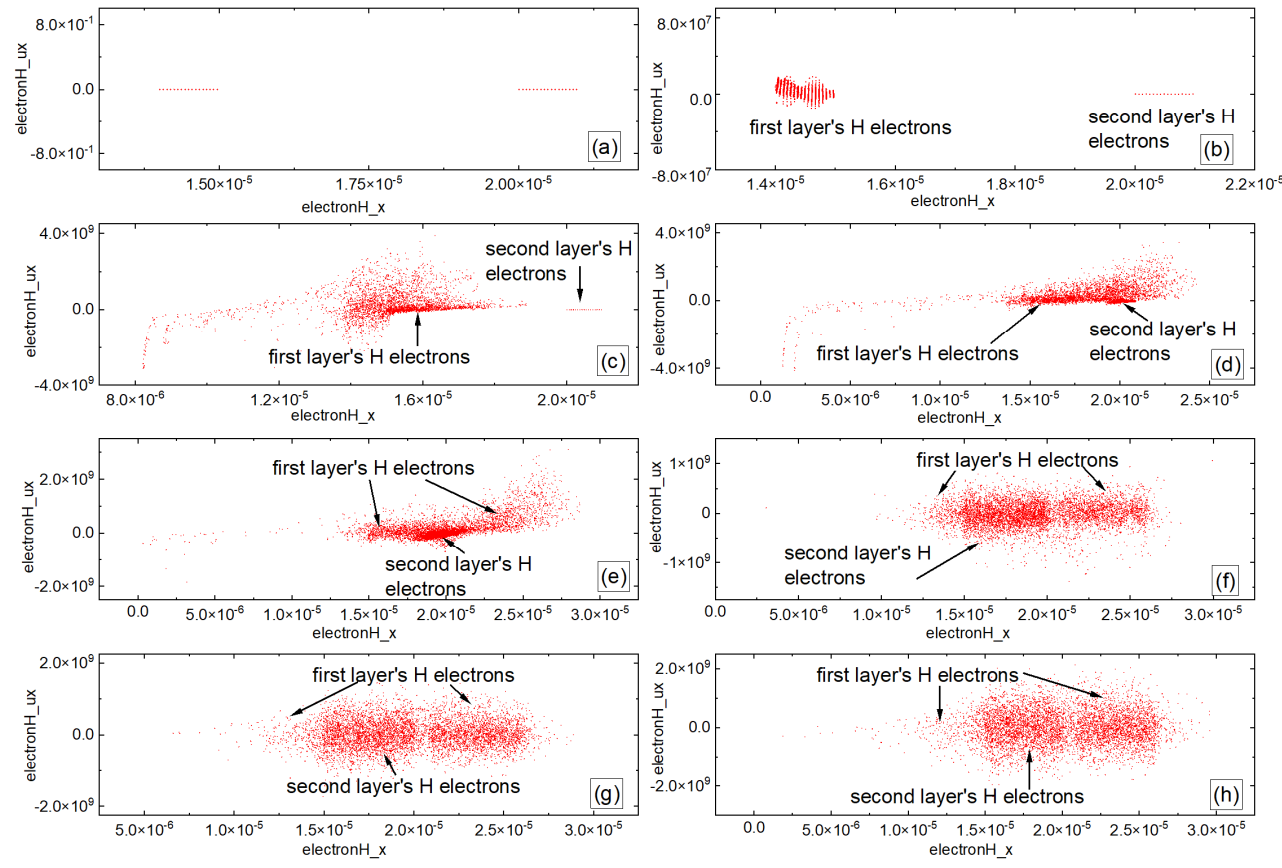

Figure 3. Two layers of hydrogen electron phase-space distribution over time with the time steps numbers being 0 (a), 11,000 (b), 15,000 (c), 18,000 (d), 20,000 (e), 26,000 (f), 34,000 (g) and 40,000 (h), respectively.
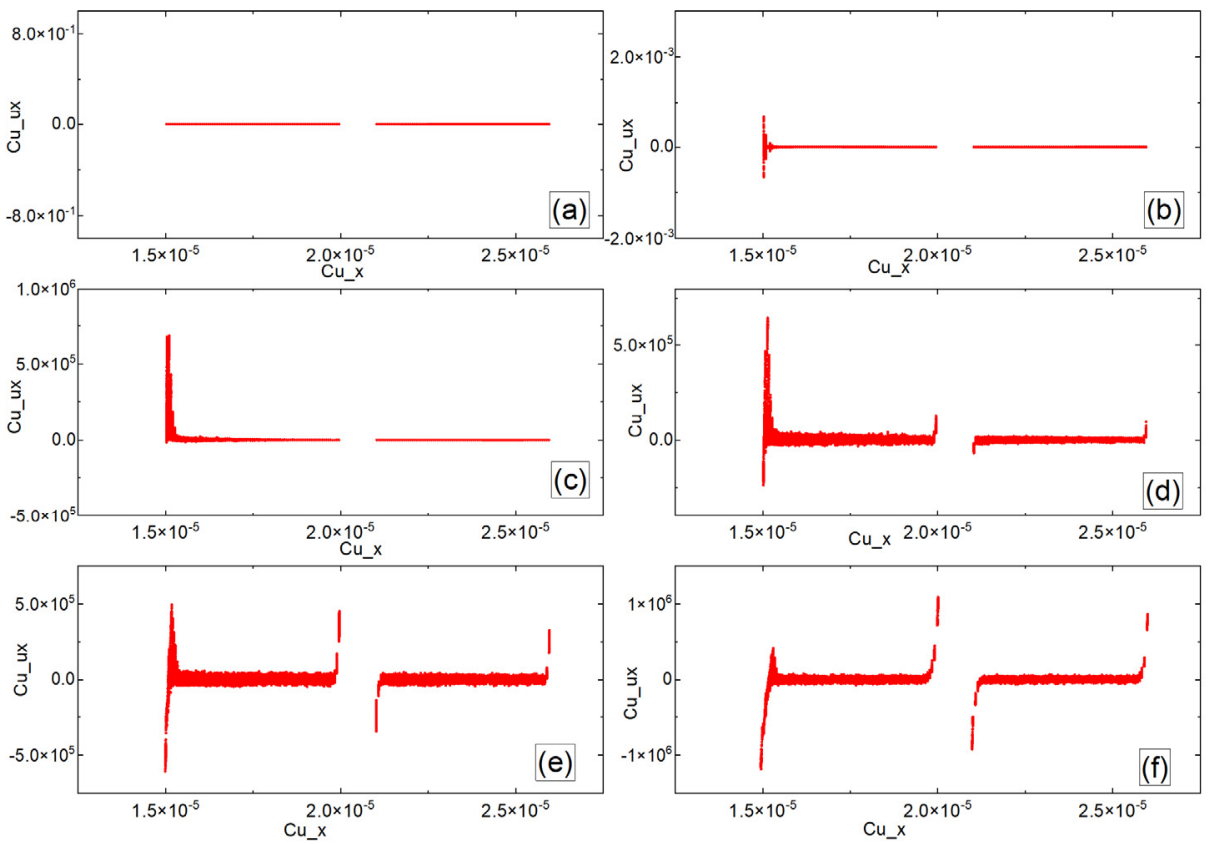

Figure 4. Two layers of copper proton phase-space distribution. From (a-f), the time steps numbers are $0,8000,16,000,24,000,32,000$, and 40,000 respectively. 


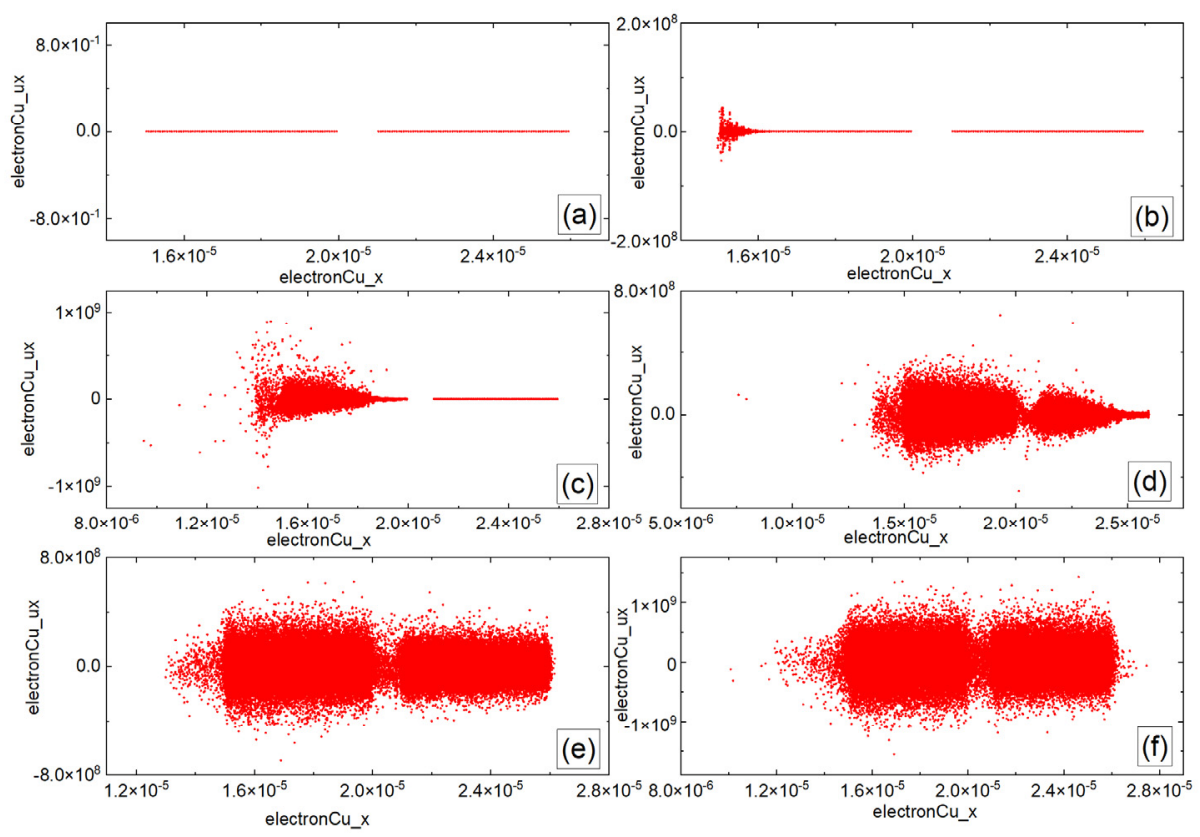

Figure 5. Two layers of copper electron phase-space distribution over time with time steps numbers are $0(\mathbf{a}), 12,000(\mathbf{b}), 16,000(\mathbf{c}), 20,000(\mathbf{d}), 27,000(\mathbf{e})$ and 39,000 (f), respectively.

\section{The Influence of the Different Thicknesses of the First Hydrogen Layer on Phase-Space Distribution under Asymmetric Structure of the Hydrogen Layer (Numerical Simulation)}

The hydrogen layer has an asymmetric structure, and the copper layer has a symmetric structure. We studied the phase-space distribution of copper electrons, copper ions, hydrogen electrons, and hydrogen protons under various widths of the first hydrogen layer. Figure 6 shows that the first hydrogen layer width was varied with 1,3, and $5 \mathrm{~nm}$, respectively. In Figure 7, we compared protons of hydrogen phase-space distribution with the time steps number being 40,000, with the first hydrogen layer being 1,3, or $5 \mathrm{~nm}$, separately. The red color plot represents the hydrogen proton phase-space distribution of the first hydrogen layer with $1 \mathrm{~nm}$, the blue color plots represent the first hydrogen layer with $3 \mathrm{~nm}$, and the yellow color plots represent the first hydrogen layer with $5 \mathrm{~nm}$. We note that the phase-space distribution of the second layer of hydrogen protons did not change with the different thicknesses of the first hydrogen layer.

Figure 8 shows the process of hydrogen electron phase-space distribution with the time steps number being 40,000 and the first hydrogen layer being 1 (red plots), 3 (blue plots), and $5 \mathrm{~nm}$ (yellow plots), respectively. It can be noted that most of the first layer of hydrogen electrons moved to the right of the second hydrogen electron layer and spread inside the second copper layer. It was noted that the second layer of hydrogen electrons moved to the left and spread inside the first copper layer. From the numerical results for the $5 \mathrm{~nm}$ hydrogen layer, most of the first layer of hydrogen electrons moved to the right of the second hydrogen layer, while some remained on the left side. For the $3 \mathrm{~nm}$ layer, fewer first layer hydrogen electrons remained on the left side. While for the $1 \mathrm{~nm}$ layer, even fewer first layer hydrogen electrons remained on the left side of the second hydrogen layer in comparison to the $3 \mathrm{~nm}$ layer. 

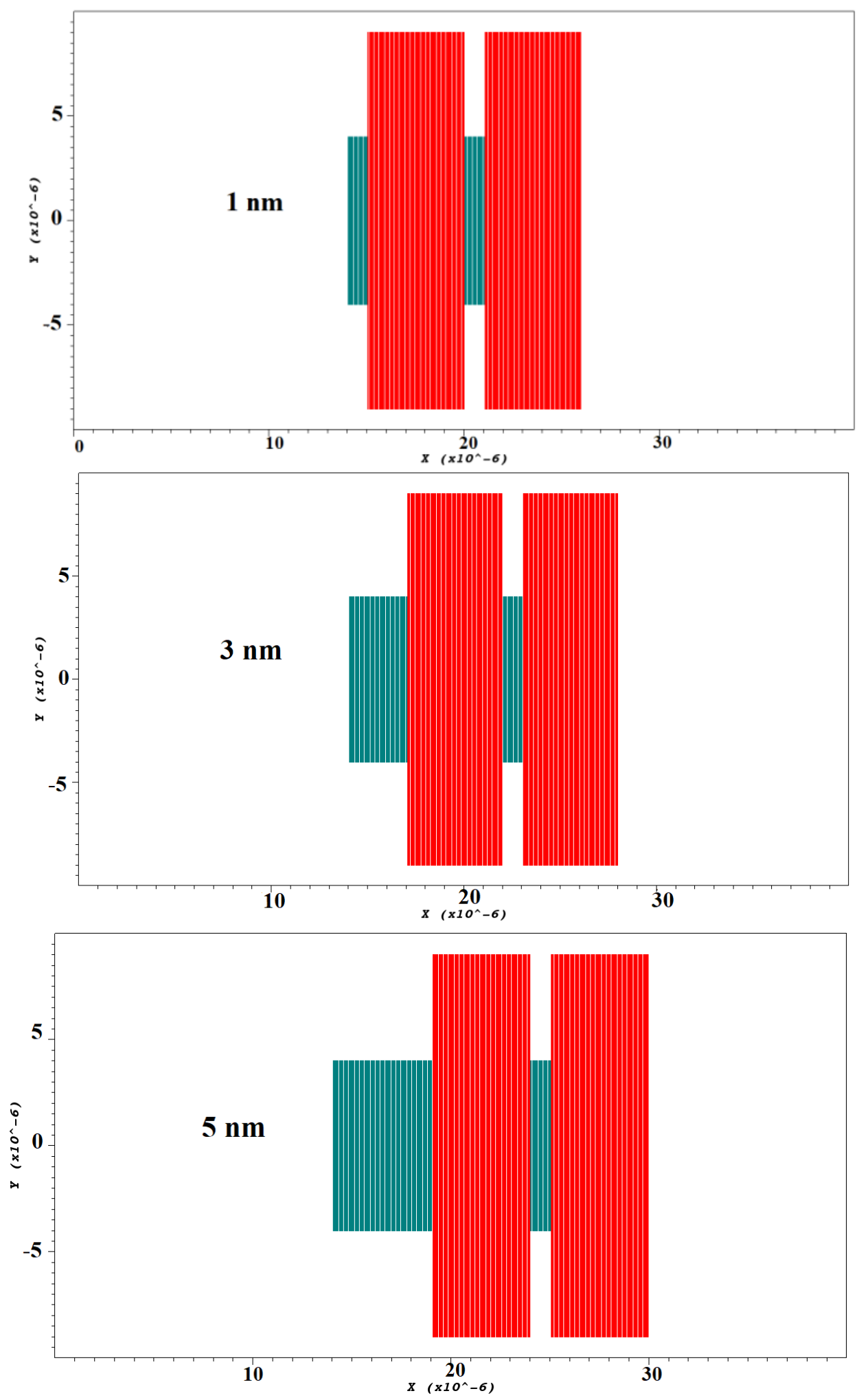

Figure 6. Differing widths of the first hydrogen layer. 


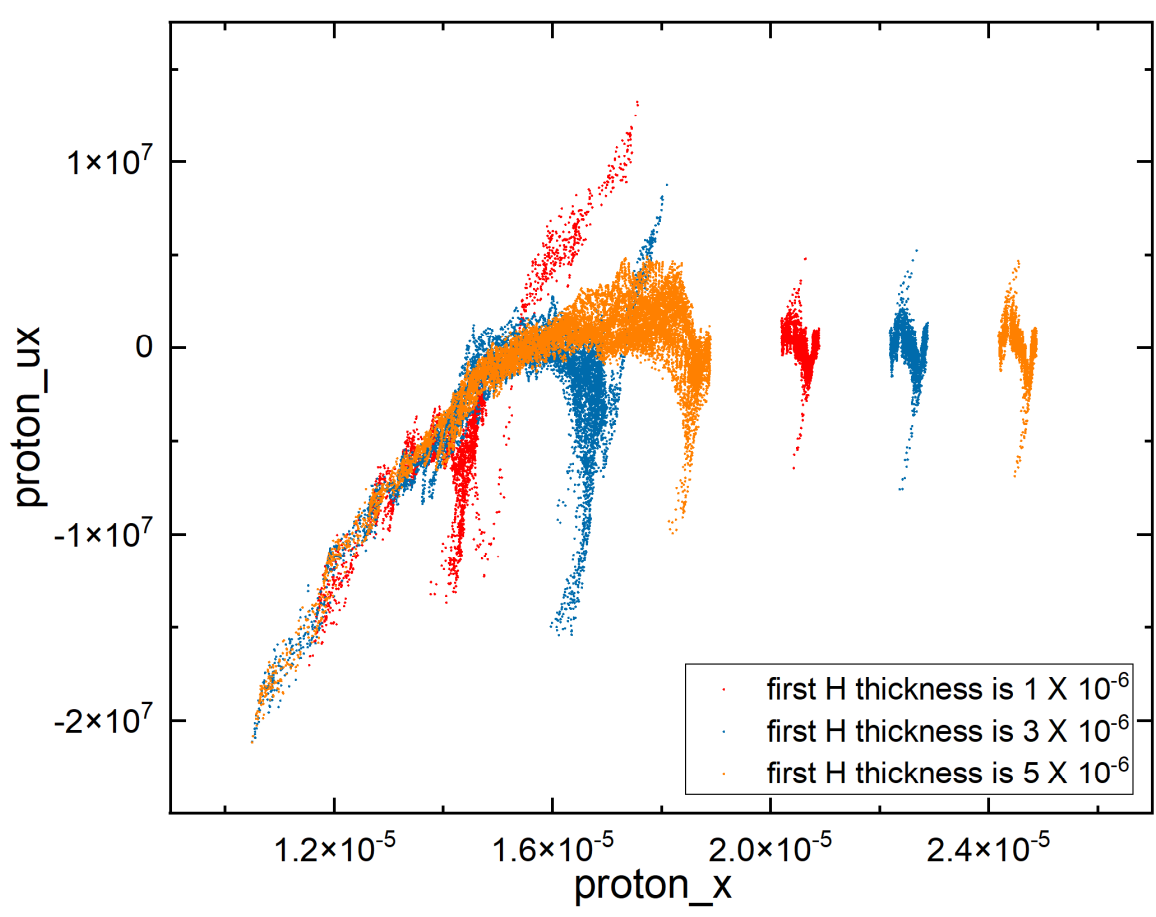

Figure 7. The comparison of hydrogen proton phase-space distribution with different first hydrogen layer widths. Red plot is the hydrogen proton phase-space distribution with first $\mathrm{H}$ thickness being $1 \mathrm{~nm}$, blue plot is it with first $\mathrm{H}$ layer being $3 \mathrm{~nm}$, and orange plot is it with first $\mathrm{H}$ layer bing $5 \mathrm{~nm}$.

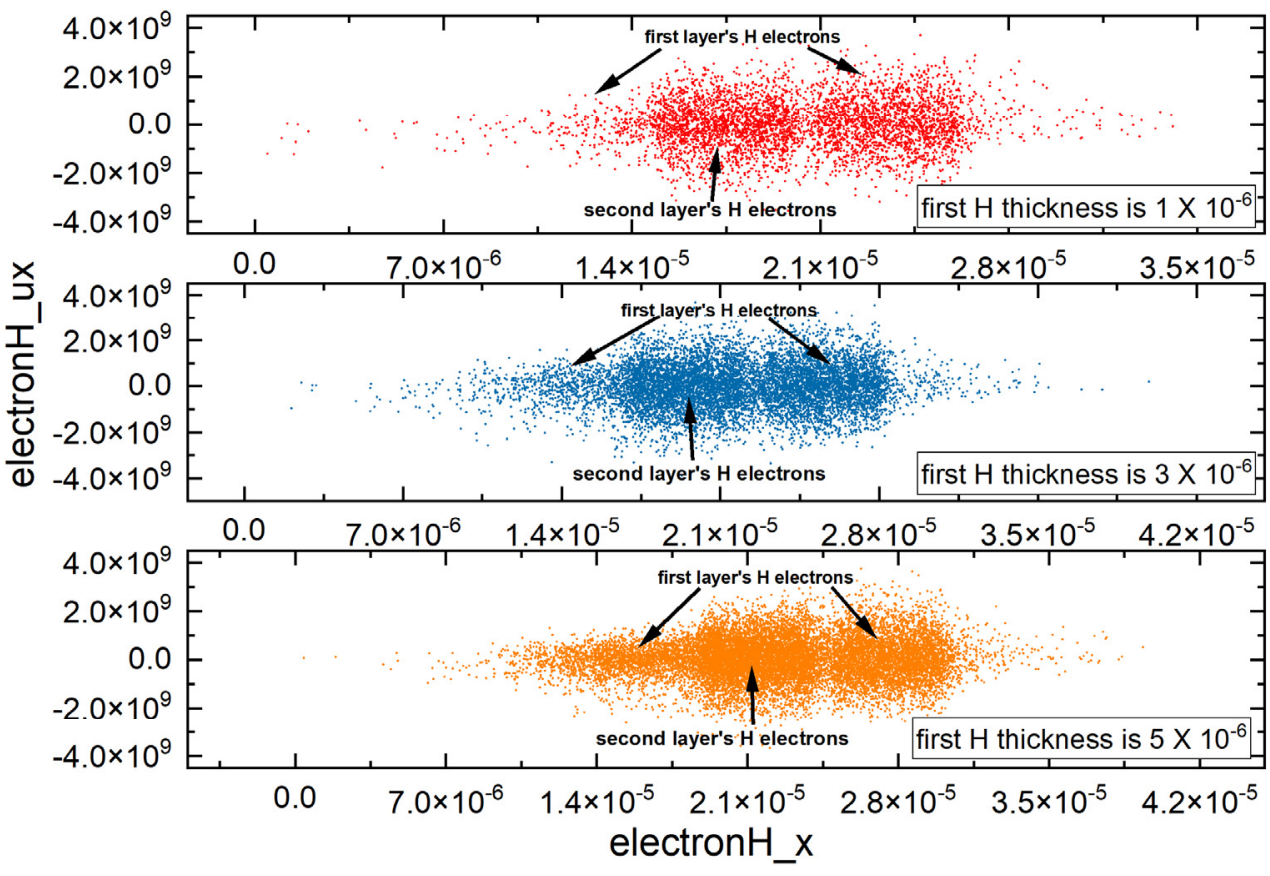

Figure 8. The comparison of hydrogen electron phase-space distribution with different first hydrogen layer widths being $1 \mathrm{~nm}, 3 \mathrm{~nm}$, and $5 \mathrm{~nm}$ respectively.

The copper ion phase-space distribution with a time step number of 40,000 in different first hydrogen layer widths is shown in Figure 9. As we can see, different hydrogen layer thickness had little influence on the acceleration of the first copper layer ions along the vertical direction, which does not influence second copper layer ions. 


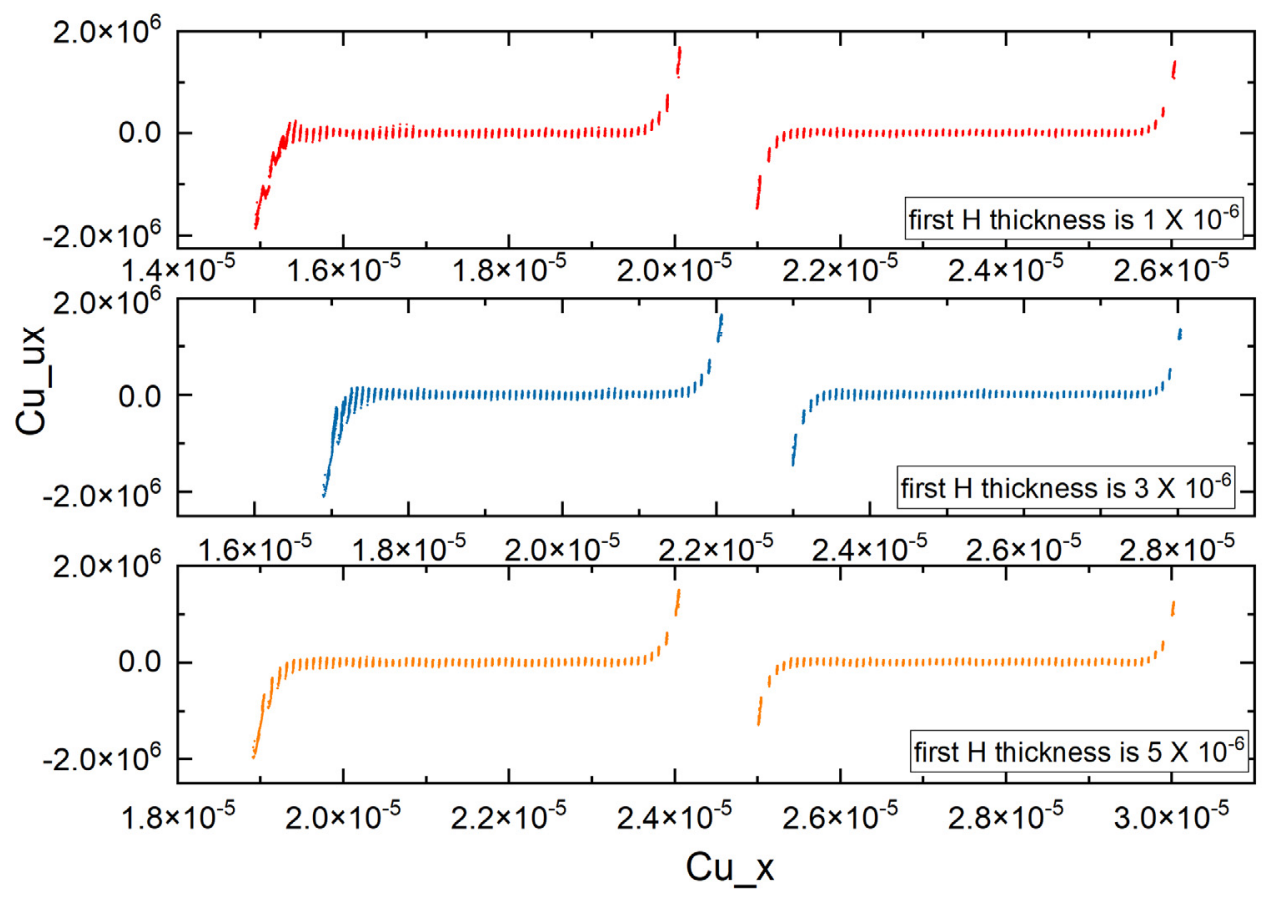

Figure 9. The comparison of copper ion phase-space with the first hydrogen layer widths being $1 \mathrm{~nm}$, $3 \mathrm{~nm}$ and $5 \mathrm{~nm}$, respectively.

The current study aims to investigate the copper electron phase-space distribution with a time step number of 40,000, as shown in Figure 10, where the red color represents the first hydrogen layer with $1 \mathrm{~nm}$, the blue color represents the first hydrogen layer with $3 \mathrm{~nm}$, and the yellow color represents the first hydrogen layer with $5 \mathrm{~nm}$. It is observed from Figure 10 that by increasing the thickness of the first hydrogen layer, the number of first copper layer electrons moving to the left direction increased. It can be seen that the second copper layer electron phase-space distribution was not influenced remarkably by the first hydrogen layer thickness.

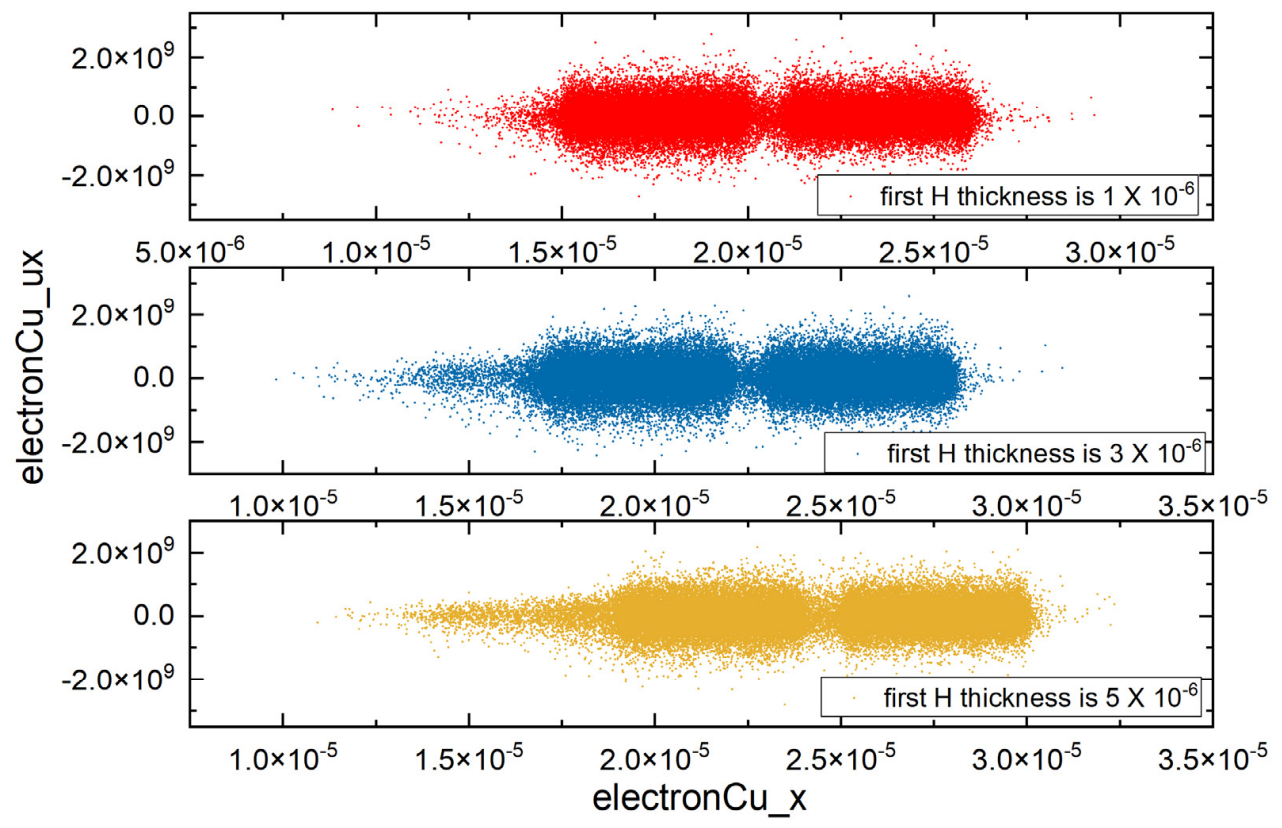

Figure 10. The comparison of copper electron phase-space distribution with the first hydrogen layer widths being $1 \mathrm{~nm}, 3 \mathrm{~nm}$ and $5 \mathrm{~nm}$, respectively. 
6. The Influence of the Different Thicknesses of the First Copper Layer on Phase-Space Distribution under Asymmetric Structure of Copper Layer (Numerical Simulation)

The influence of varied first copper layer thicknesses on the phase-space distribution of copper electrons, copper ions, hydrogen electrons, and hydrogen protons was also investigated. Fixing other layer thicknesses, the first copper layers with different thicknesses (1, 3 , and $5 \mathrm{~nm}$ ) are shown in Figure 11. The Copper layer has an asymmetric structure.
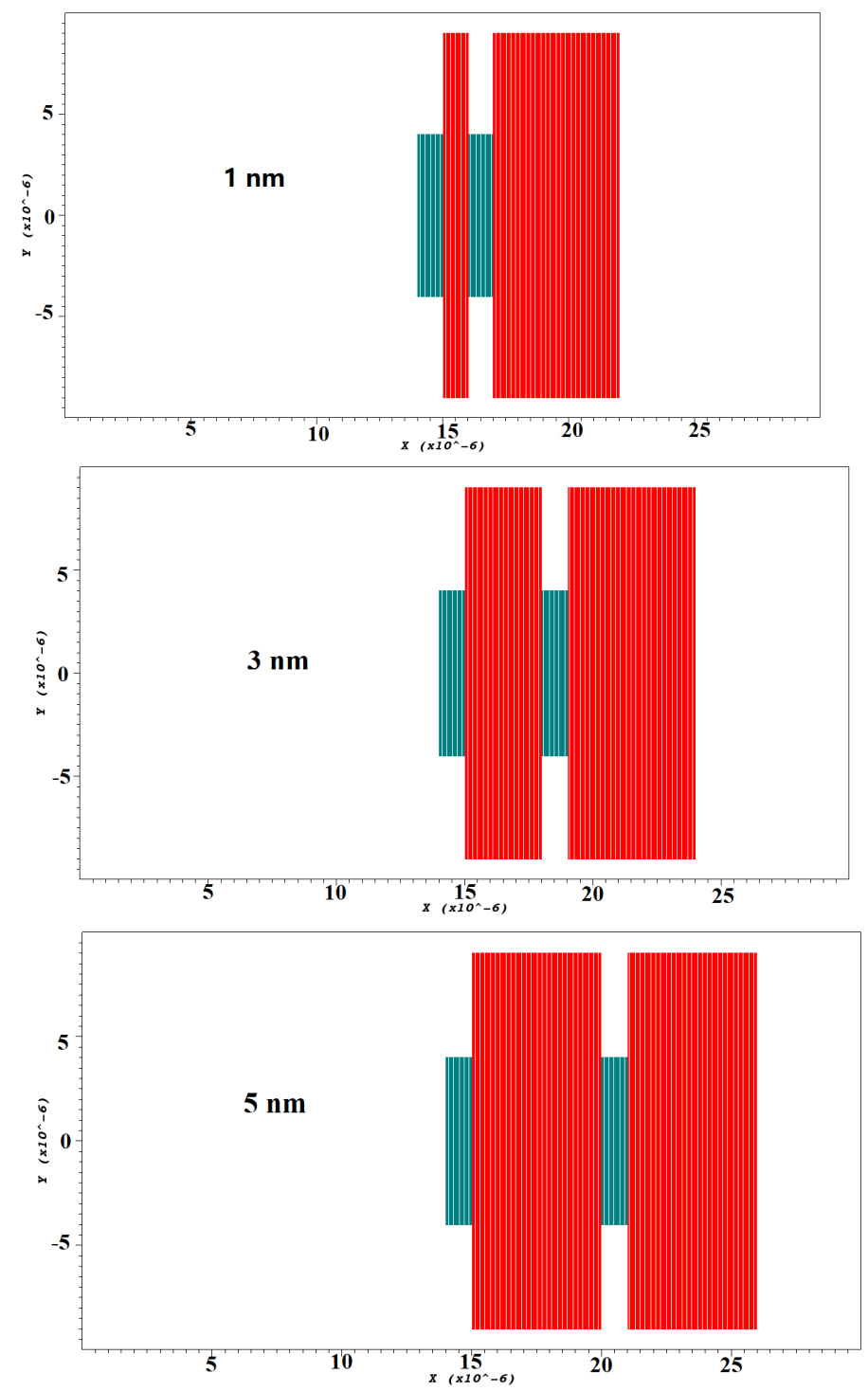

Figure 11. Different widths of first copper layers.

In Figure 12, we compare the results of the hydrogen proton phase-space distribution with a time step number of 40,000 with the first copper layer of respective 1, 3, and $5 \mathrm{~nm}$. The red color represents hydrogen proton phase-space distribution with the first copper layer width of $1 \mathrm{~nm}$, the blue color is the first copper layer with $3 \mathrm{~nm}$, and the yellow color is the first copper layer with $5 \mathrm{~nm}$. We can observe that the phase-space distribution of the first hydrogen proton nearly had no change among different first copper layer widths. It can be concluded that the first copper layer's thickness cannot influence the first hydrogen layer's proton acceleration in the horizontal and vertical directions. It can also be noted that the acceleration of the second layer of hydrogen protons was greater with the first copper layer being $5 \mathrm{~nm}$ than it with the first copper layer being $1 \mathrm{~nm}$ in the vertical direction, while the acceleration of the second layer of hydrogen protons was greater with 
the first copper layer being $1 \mathrm{~nm}$ than it with the first copper layer being $5 \mathrm{~nm}$ in the horizontal direction.

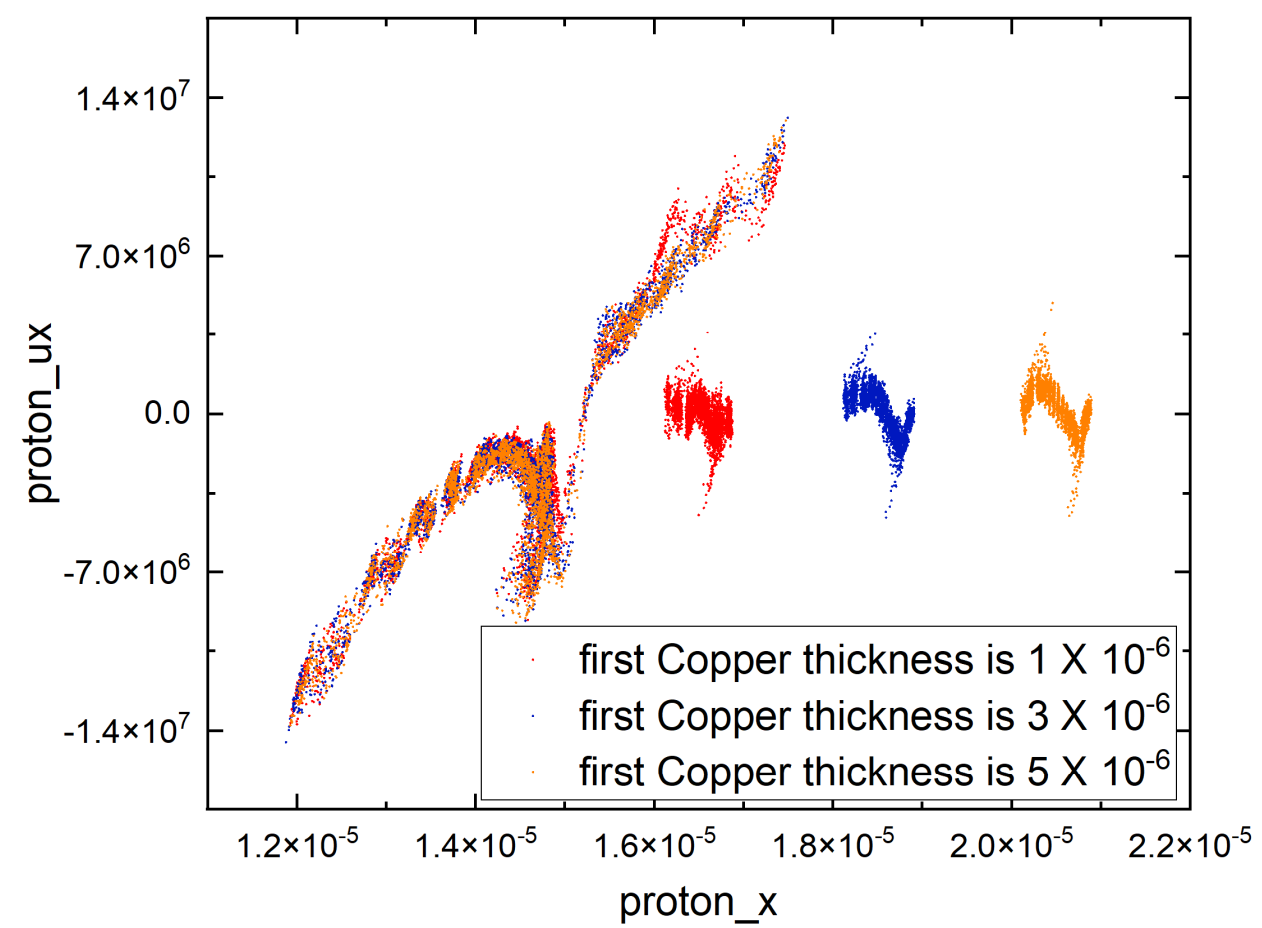

Figure 12. The comparison of hydrogen proton phase-space distribution in different first copper layer widths. Red one is first copper layer being $1 \mathrm{~nm}$, blue one is first copper layer being $3 \mathrm{~nm}$, and orange one is first copper layer being $5 \mathrm{~nm}$.

We investigated the hydrogen electrons phase-space distribution with the time step number of 40,000 among different first copper layer thicknesses in Figure 13. We found that behaviors of the first hydrogen layer electrons were hardly influenced by the different widths of the first copper layer; even most first hydrogen layer electrons were pushed to the right side of the second hydrogen layer electrons. Nearly the same percentage of first hydrogen layer electrons remained in the left part in differing widths of the first copper layer. It is necessary to note that most first hydrogen layer electrons spread into the second copper layer. However, the second hydrogen layer's electrons were influenced remarkably by the different thicknesses of the first copper layer. Most of the second hydrogen layer's electrons moved and spread inside the first copper layer. Therefore, it can be noted that the width of the second hydrogen layer's electrons was 1,3 , or $5 \mathrm{~nm}$ when the first copper width was 1,3 , or $5 \mathrm{~nm}$, respectively.

Figure 14 shows the copper ion phase-space distribution among different first copper layer thicknesses. It can be noted that only the first copper layer ion phase-space distribution was different due to the differing first copper layer thickness, while the second copper layer ions were not influenced by varying the first copper layer thickness.

The results are presented for the copper electron phase-space distribution following the variation of the first copper layer width in Figure 15. Based on the simulation results, we can see that the first copper layer electron phase-space distribution was different due to the different first copper layer widths, while the second copper layer electron phase-space distribution was constant when the first copper layer's width was varied. 


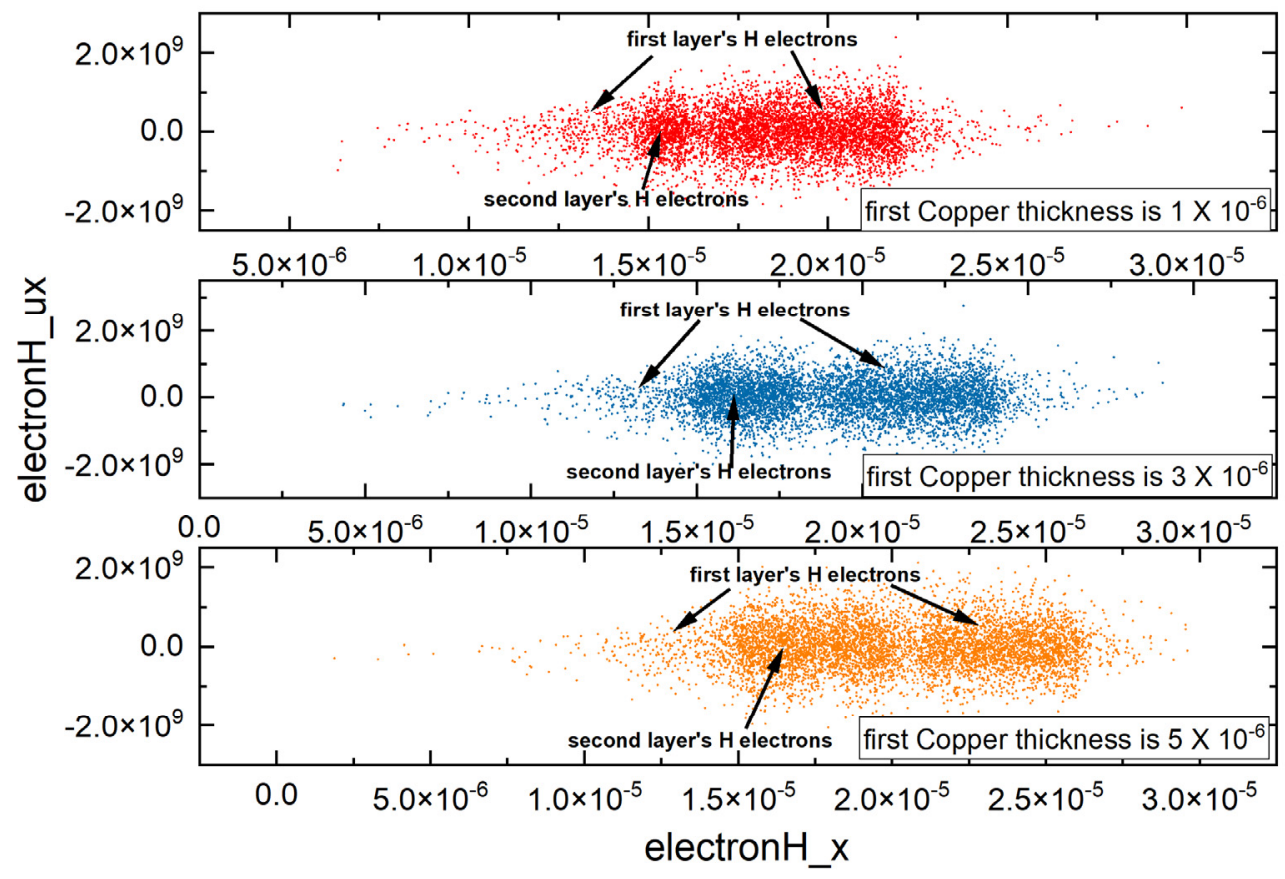

Figure 13. The comparison of hydrogen electron phase-space distribution among different first copper layer widths. Red plot is hydrogen electron phase-space distribution with first copper layer being $1 \mathrm{~nm}$. Blue plot is it with first copper layer being $3 \mathrm{~nm}$. And orange one is it with $5 \mathrm{~nm}$ first copper layer.

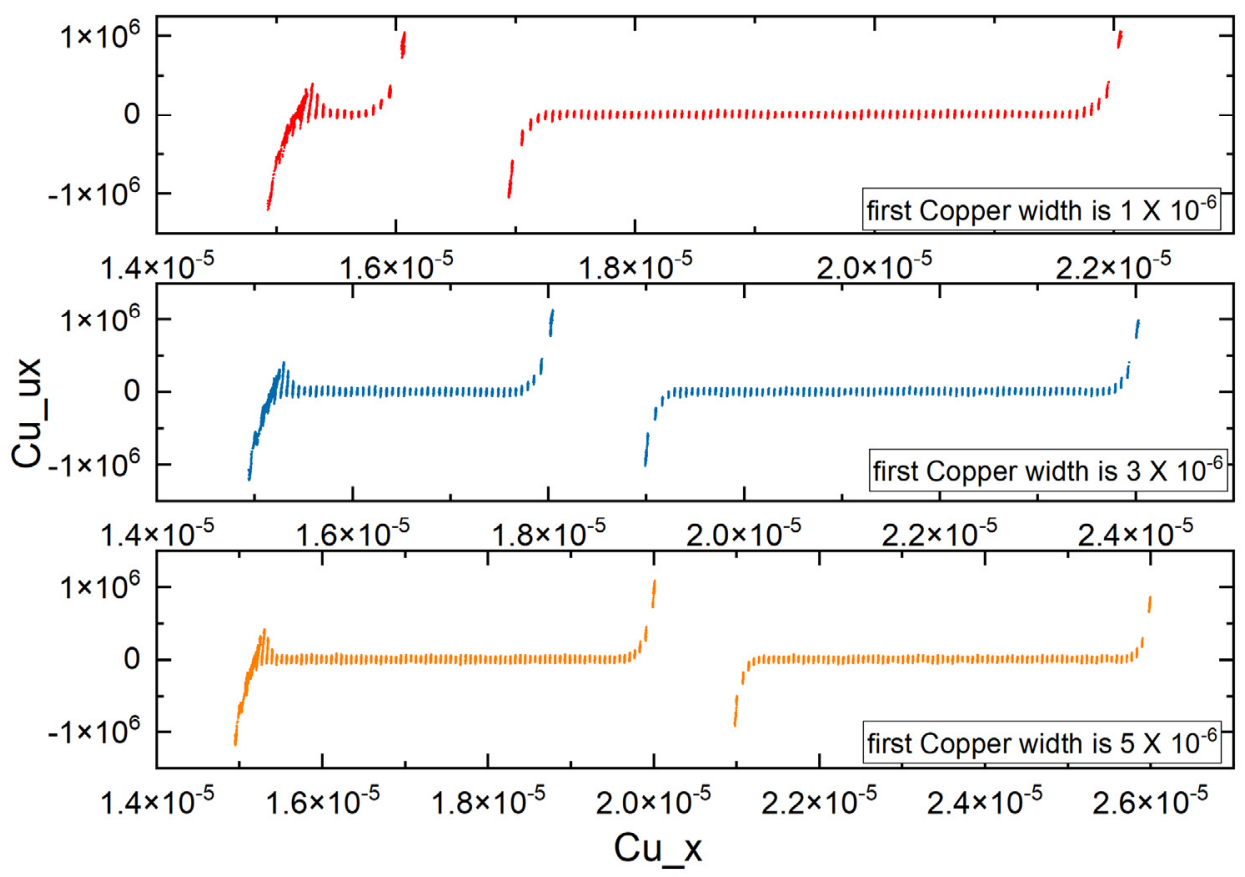

Figure 14. The comparison of copper proton phase-space distribution with different first copper layer widths. Red one is copper proton phase-space distribution with first copper width being $1 \mathrm{~nm}$. Blue one is it with first copper width being $3 \mathrm{~nm}$. And orange one is it with $5 \mathrm{~nm}$ first copper layer width. 


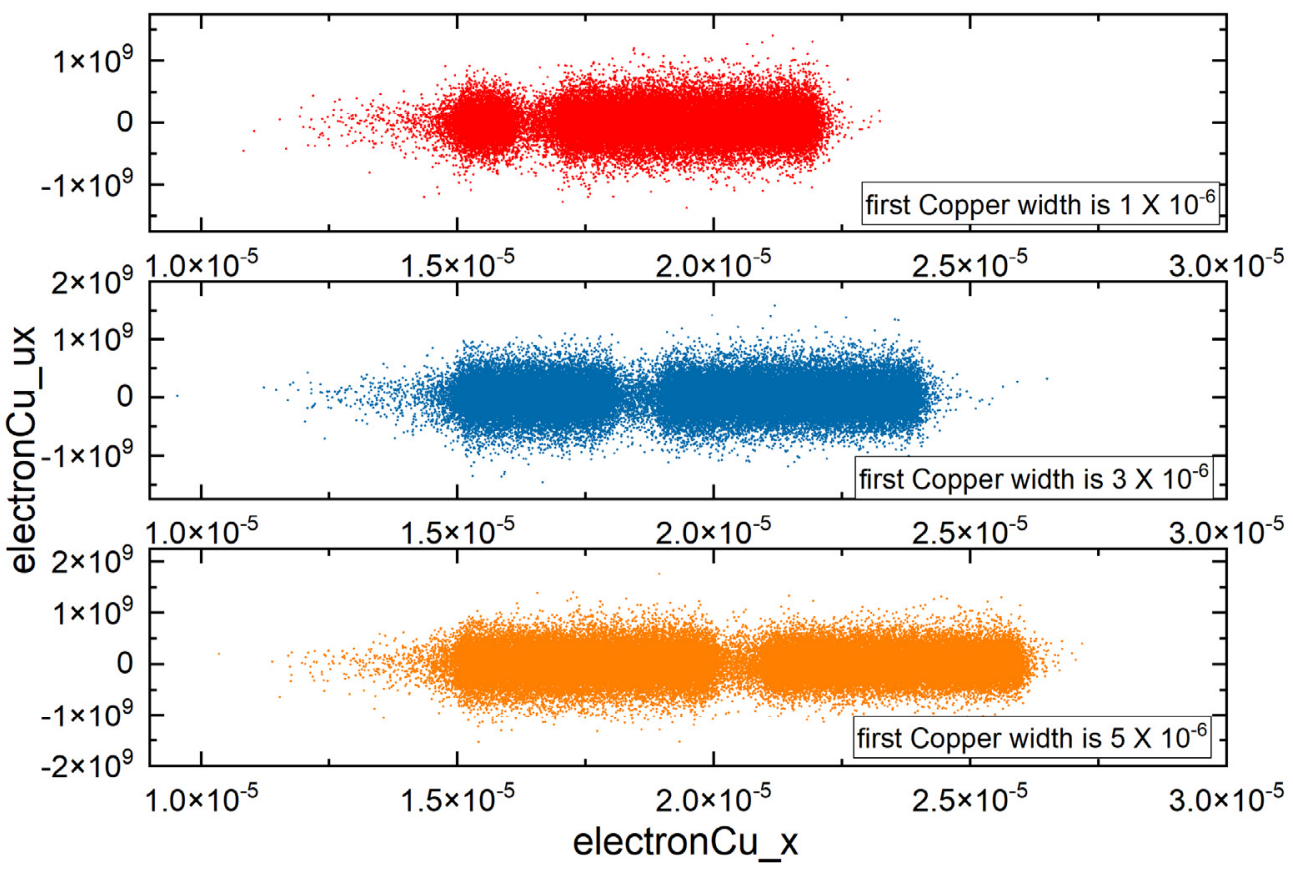

Figure 15. The comparison of copper electron phase-space distribution with different first copper layer widths. Red plot, blue plot, and orange plot are copper electron phase-space distribution with first cop-per layer width being $1 \mathrm{~nm}, 3 \mathrm{~nm}$, and $5 \mathrm{~nm}$, respectively.

\section{Conclusions}

The super-strong and ultra-short laser pulses interacting with the multiple copper layers covered with multiple hydrogen layers to accelerate the ion mechanism was studied by simulation. We also theoretically investigated the plasma phase-space distribution by varying the thickness of the first hydrogen layer and the first copper layer. The theoretical simulation results showed that when the ultra-intense laser pulses irradiated the multiple copper layers covered with multiple hydrogen layers, different plasma phase-space distributions showed different phenomena under the differing widths of the first hydrogen layer or first copper layer. We compared the phase-space distribution of copper electrons, copper ions, hydrogen electrons, and hydrogen protons when the first hydrogen layer was 1,3 , or $5 \mathrm{~nm}$. Meanwhile, the phase-space distribution of four plasma was also observed when the first copper layer was 1,3 , or $5 \mathrm{~nm}$. The thickness of the first hydrogen layer exerted a significant influence on some plasma velocity and acceleration distributions, while other plasma velocity and acceleration distributions were not influenced. The thickness of the first copper layer was the same. These simulation research results are important supplements to the studies of the interaction between ultra-intense ultra-short pulses and plasma.

Author Contributions: Software, data curation and writing—original draft preparation, F.F.; supervision and project administration, G.L. Both authors have read and agreed to the published version of the manuscript.

Funding: This research was funded by THE OPEN FUND OF KEY LABORATORY OF ICING AND AN-TI/DE-ICING, grant No. IADL20200405; This research was also funded by FUND OF “ACADEMIC BACKBONE” OF NORTHEAST AGRICULTURAL UNIVERSITY, grant No.20XG20.

Institutional Review Board Statement: Not applicable.

Informed Consent Statement: Not applicable.

Data Availability Statement: The research data have been included in the article. 
Conflicts of Interest: I would like to declare on behalf of my co-authors that the work described was original research that has not been published previously, and not under consideration for publication elsewhere, in whole or in part, no conflict of interest exits in the submission of this manuscript, and manuscript is approved by all authors for publication.

\section{References}

1. Bulanov, S.V.; Yamagiwa, M.; Esirkepov, T.Z.; Dylov, D.V.; Kamenets, F.F.; Knyazev, N.S.; Koga, J.K.; Kando, M.; Ueshima, Y.; Saito, K.; et al. Electron bunch acceleration in the wake wave breaking regime. Plasma Phys. Rep. 2006, 32, 263-281. [CrossRef]

2. Pukhov, A.; Meyer-ter-Vehn, J. Laser Wake Field Acceleration: The Highly Non-Linear Broken-Wave Regime. Appl. Phys. B 2002, 74, 355-361. [CrossRef]

3. Faure, J.; Glinec, Y.; Pukhov, A.; Kiselev, S.L.; Gordienko, S.N.; Lefebvre, E.; Rousseau, J.-P.; Burgy, F.; Malka, V. A laser-plasma accelerator producing monoenergetic electron beams. Nat. Cell Biol. 2004, 431, 541-544. [CrossRef] [PubMed]

4. Geddes, C.G.R.; Toth, C.; Van Tilborg, J.J.; Esarey, E.; Schroeder, C.; Bruhwiler, D.; Nieter, C.; Cary, J.; Leemans, W.P. High-quality electron beams from a laser wakefield accelerator using plasma-channel guiding. Nat. Cell Biol. 2004, 431, 538-541. [CrossRef] [PubMed]

5. Peter, M.; Bruhwiler, D.L.; Cary, J.R.; Dimitrov, D.A. Ion Acceleration and Wave Generation by Overdense Laser-Plasma Interaction. AIP Conf. Proc. 2004, 737, 621-627.

6. Wolowski, J.; Badziak, A.J.; Czarnecka, S.; Gammino, J.; Láska, K.L.; Mezzasalma, A.; Parys, P.; Pfeifer, M.; Rohlena, K. Interaction of High-Energy Laser Pulses with Plasmas of Different Density Gradients. In Proceedings of the 32nd EPS Conference on Plasma Physics 2005 (EPS 2005), Tarragona, Spain, 27 June-1 July 2005; Volume 29C.

7. Hora, H.; Badziak, J.; Boody, F.; Höpfl, R.; Jungwirth, K.; Králikova, B.; Krása, J.; Láska, L.; Parys, P.; Perina, V.; et al. Effects of ps and ns laser pulses for giant ion source. Opt. Commun. 2002, 207, 333-338. [CrossRef]

8. Wang, G.H.; Wang, X.F.; Dong, K.G. Ultra-short ultra-intense laser guiding and its influence on electron acceleration. Acta Phys. Sin. 2012, 61, 165201.

9. Yang, Z.; Yuan, P.; Li, Z.; Yang, Z. L-shell X-ray emission measurement of Au plasma produced by intense femtosecond laser. High Power Laser Part Beams 2012, 24, 1896-1900. [CrossRef]

10. Lifschitz, A.; Sylla, F.; Kahaly, S.; Flacco, A.; Veltcheva, M.; Sanchez-Arriaga, G.; Lefebvre, E.; Malka, V. Ion acceleration in underdense plasmas by ultra-short laser pulses. New J. Phys. 2014, 16, 033031. [CrossRef]

11. Huang, L.G.; Bussmann, M.; Kluge, T.; Lei, L.; Yu, W.; Cowan, T.E. Ion heating dynamics in solid buried layer targets irradiated by ultra-short intense laser pulses. Phys. Plasmas 2013, 20, 093109. [CrossRef]

12. Tatomirescu, D.; Popescu, A.; d'Humieres, E.; Vizman, D. Numerical Simulation of Laser Ion Acceleration at Ultra High Intensity. AIP Conf. Proc. 2017, 1796, 020013.

13. Tatomirescu, D.; d'Humieres, E.; Vizman, D. Improving the particle beam characteristics resulting from laser ion acceleration at ultra high intensity through target manipulation-Numerical modeling. AIP Conf. Proc. 2017, 1916, 030002.

14. Ma, Y.; Zhao, J.; Li, Y.; Li, D.; Chen, L.; Liu, J.; Dann, S.J.D.; Ma, Y.; Yang, X.; Ge, Z.; et al. Ultrahigh-charge electron beams from laser-irradiated solid surface. Proc. Natl. Acad. Sci. USA 2018, 115, 6980-6985. [CrossRef] [PubMed]

15. Fiore, G.; Catelan, P. On cold diluted plasmas hit by short laser pulses. Nucl. Inst. Methods Phys. Res. A 2018, 909, 41. [CrossRef]

16. Ma, W.; Kim, I.J.; Yu, J.; Choi, I.W.; Singh, P.K.; Lee, H.W.; Sung, J.H.; Lee, S.K.; Lin, C.; Liao, Q.; et al. Laser Acceleration of Highly Energetic Carbon Ions Using a Double-Layer Target Composed of Slightly Underdense Plasma and Ultrathin Foil. Phys. Rev. Lett. 2019, 122, 014803. [CrossRef] [PubMed]

17. Abuduresuli, A.; Amat, N.; Okada, T. Numeral Simulation of Energetic Ion Generation. J. Xinjiang Univ. $2007,24,2$.

18. Ma, Y.; Chang, W.; Yin, Y.; Cao, L.; Yue, Z. Particle Simulation on Plasma Bubble and Energetic Ion Generation Produced by Laser. Chin. J. High Presure Phys. 2002, 16, 147-151. 